Théologiques

Théologiques

\title{
La théorie de la libération dans le contexte socio-économique de l'Amérique latine : économie et théologie ou l'irrationalité du « rationalisé »
}

\section{Frank J. Hinkelammert}

Volume 3, numéro 2, octobre 1995

Crise du travail, crise de civilisation

URI : https://id.erudit.org/iderudit/602428ar

DOI : https://doi.org/10.7202/602428ar

Aller au sommaire du numéro

\section{Éditeur(s)}

Faculté de théologie de l'Université de Montréal

ISSN

1188-7109 (imprimé)

1492-1413 (numérique)

Découvrir la revue

Citer cet article

Hinkelammert, F. J. (1995). La théorie de la libération dans le contexte

socio-économique de l'Amérique latine : économie et théologie ou

l'irrationalité du " rationalisé ». Théologiques, 3(2), 115-151.

https://doi.org/10.7202/602428ar

\section{Résumé de l'article}

Cet article retrace, à travers l'expérience personnelle qu'en a connue l'auteur, la genèse, la trajectoire et le rôle fondamental qu'a joué la question de l'économie au sein de la théologie de la libération. C'est à partir de l'articulation originale de cette théologie à l'économie qu'il rend compte de l'hostilité quelle a suscitée de la part de l'orthodoxie ecclésiale et théologique et de la part de l'« orthodoxie » du capitalisme néo-libéral. Il décortique longuement la « théologie » capitaliste qui tente maintenant de se substituer à la théologie de la libération après l'avoir vainement dénoncée. L'article se termine sur une critique théologique acérée de l'absolutisation de la « loi ", de l'anti-utopisme et de la sacrificialité inhérents au néo-libéralisme actuel, qualifié de totalitaire. 
Theologiques 3/2 (1995) 115-151.

\title{
La théologie de la libération dans le contexte socio-économique de I'Amérique latine : économie et théologie ou l'irrationalité du « rationalisé » 1
}

\author{
Frank J. HINKELAMMERT \\ Directeur du Departamento Ecuménico de Investigaciones (DEI) \\ San José, Costa Rica
}

\section{RÉSUMÉ}

Cet article retrace, à travers l'expérience personnelle qu'en a connue l'auteur, la genèse, la trajectoire et le rôle fondamental qu'a joué la question de l'économie au sein de la théologie de la libération. C'est à partir de l'articulation originale de cette théologie à l'économie qu'il rend compte de l'hostilité qu'elle a suscitée de la part de l'orthodoxie ecclésiale et théologique et de la part de l'a orthodoxie * du capitalisme néo-libéral. Il décortique longuement la a théologie * capitaliste qui tente maintenant de se substituer à la théologie de la libération après l'avoir vainement dénoncée. L'article se termine sur une critique théologique acérée de l'absolutisation de la "loi ", de l'anti-utopisme et de la sacrificialité inhérents au néo-libéralisme actuel, qualifié de totalitaire.

Je désirerais, dans les pages qui suivent, proposer une lecture de la théologie de la libération à partir de l'expérience que j'en ai vécue comme participant de son développement. Je ne prétends pas faire l'histoire de cette théologie. Cela conduirait à une analyse beaucoup plus nuancée et probablement plus impartiale que ce que je peux offrir. J'essaierai plutôt de présenter ce qui a été ma préoccupation et celle des compagnons et

1 Traduit de l'espagnol par Madeleine Perreault en collaboration avec Michel Beaudin. 
compagnes avec lesquels j'ai travaillé depuis 1976 au DEI, et qui nous a amenés à nous sentir partie prenante de ce courant de pensée dans l'Amérique latine d'aujourd'hui. Il s'agit précisément de la thèse qui veut que toute théologie de la libération doive se développer dans la discussion théorique du rapport entre l'économie et théologie. C'est pourquoi le point de vue développé dans cet article sera axé sur cette problématique.

\section{La théologie de la libération comme théologie concrète}

La théologie de la libération est une théologie concrète, une théologie historique. Il est donc approprié de s'interroger sur le contexte historique de cette théologie. Son développement couvre les trois dernières décades d'une histoire latino-américaine très agitée. Nous allons, par conséquent, avoir affaire, avec la théologie de la libération, à une théologie qui réfléchit sur cette histoire dont elle est consciente de faire partie, qui la reflète et qui la ré-élabore à nouveau.

En tant que théologie concrète, la théologie de la libération s'inscrit dans la société latino-américaine. Communautés de base, mouvements populaires et partis politiques sont des lieux-clés à partir desquels cette théologie se développe et s'insère donc dans l'histoire concrète de l'Amérique latine. Elle ne fait pas que parler dans ces lieux concrets, mais c'est aussi à partir d'eux qu'elle réfléchit sur la situation historique pour se développer en tant que théologie. C'est en raison de cela que ses analyses sont étroitement liées aux théories des sciences sociales. Elle ne peut déduire ses analyses concrètes de ses positions théologiques, mais d'autre part, ses positions théologiques restent inséparables du résultat de ses analyses concrètes.

La théologie de la libération n'est toutefois pas pour autant une science sociale; il s'agit bien d'une théologie. Articulée étroitement à ses analyses concrètes de la situation historique, cette théologie se développe comme une dimension théologique de cette situation historique. Elle en court d'autant le risque de se tromper. Elle ne possède pas de vérités absolues spécifiques a priori. Pour employer des termes très souvent utilisés par les théologiens de la libération, il s'agit de l'unique orthodoxie chrétienne possible, une orthopraxie. Le christianisme lui-même est né en tant qu'orthopraxie et non comme un système clos d'affirmations dogmatiques vides. Trouver la praxis pertinente à la situation, voilà le projet de cette théologie. C'est pour cela qu'elle ne cesse de se développer au fur et à mesure où les problèmes changent et où les théologiens acquièrent des connaissances nouvelles pour leur faire face. C'est une théologie vivante. 
Toutefois, une théologie peut s'énoncer en tant que telle avant même d'entrer dans l'analyse concrète et dans l'orthopraxie correspondante. En tant que théologie, elle précède la praxis. Mais en précédant la praxis, elle forme un ensemble de croyances vides. L'existence de Dieu, son caractère trinitaire, la rédemption, etc., professés comme des actes d'une foi indépendante de son insertion historique et concrète, ne sont que de abstractions vides formant un dogmatisme sans contenu. Le problème de la théologie de la libération, ce n'est pas de les nier, mais plutôt de se questionner sur leur signification. Par conséquent, elle ne se demandera pas : "Dieu existe-t-il? " mais plutôt : « où Dieu est-il présent? » et " comment agit-il? » Le point de départ de la théologie de la libération est donc la question du lieu concret et historique où Dieu se révèle.

La théologie de la libération naît de la réponse qu'elle donne à cette question. Cette réponse se trouve dans ce que ces théologiens appellent "l'option pour le pauvre". Cetre option pour le pauvre, c'est l'option de Dieu; mais c'est en même temps l'option des êtres humains dès qu'ils veulent se libérer. La libération dont il est question, c'est donc la libération du pauvre, mais pas comme l'action d'autres personnes qui auraient le devoir de libérer le pauvre considéré comme objet. Sans cette reconnaissance mutuelle entre sujets, dont la pauvreté apparaît comme la négation, il n'y a pas d'option pour le pauvre. Les sujets humains ne peuvent se reconnaître mutuellement sans qu'ils se reconnaissent comme des êtres corporels et soumis à des nécessités naturelles. La pauvreté est une négation vivante de cette reconnaissance. Bien sûr, du point de vue des théologiens de la libération, l'être humain ne peut se libérer ni devenir vraiment libre sans cette reconnaissance mutuelle entre sujets. Ainsi, le pauvre, comme sujet qui entre dans cette relation de reconnaissance, devient le lieu où se décide si cette reconnaissance sera effective ou non. Pat conséquent, l'autre aspect de la reconnaissance mutuelle des sujets humains comme des êtres liés par nature à des besoins, c'est l'option pour le pauvre $^{2}$.

2 La philosophie de Lévinas est l'une des sources de cette manière de penser. Voir: Emmanuel LÉVINAS, Totalidad e infinito. Ensayo sobre la exterioridad. Salamanca, Ediciones Sígueme, 1977; De otro modo de ser, o más allá de la esencia. Salamanca, Ediciones Sígueme, 1987. Dans un livre publié plus tard, Lévinas résume très justement cette position en parlant de l'amour du prochain: "Que signifie "comme toi-même »? Buber et Rosenzweig étaient ici bien embarrassés par la traduction. Ils se sont dir : « comme toi-même », cela ne signifie-t-il pas qu'on s'aime le plus soi-même? Au lieu de la traduire en accord avec vous, ils ont traduit : « Aime ton prochain, il est comme toi $»$. Mais, si on consent déjà à séparer le dernier mot du verset 
Donc, chez le pauvre, on constate l'absence de cette reconnaissance mutuelle entre les êtres humains. Mais, selon la théologie de la libération, Dieu est présent là où cette reconnaissance a lieu. Le fait que cette reconnaissance n'ait pas lieu révèle une relation humaine où Dieu est absent. L'existence du pauvre témoigne de l'existence d'une société sans Dieu, qu'elle croie de manière explicite en Dieu ou non. Cette absence de Dieu, néanmoins, se manifeste là où quelqu'un crie. L'absence de Dieu est présente dans le pauvre. Le pauvre est présence du Dieu absent. Il s'agit, sous le mode de la visibilité, d'un cas de théologie négative dans laquelle la présence de Dieu - une présence effective - est révélée par l'absence, une absence qui crie, et par le dénuement. En vivant l'absence de Dieu, on perçoit sa présence en accomplissant sa volonté. Par conséquent, on ne peut concevoir une présence de Dieu qui nierait l'option pour le pauvre, cette option ne fût-elle qu'implicite. Cependant, comme option, il faut qu'elle soit là. Donc, la présence de Dieu n'est pas dans tel ou tel être, mais plutôt dans une relation sociale entre les êtres humains. En étant cette reconnaissance mutuelle entre les sujets qui n'exclut personne, Dieu est présent et son absence est surmontée. Mais son absence revient dès que cette reconnaissance se perd.

La théologie de la libération naît comme théologie concrète à partir de cette réflexion, même si elle connaît des expressions différentes selon les divers théologiens. Elle a donc ainsi un lieu à partir duquel elle peut interpréter la réalité historique. C'est un lieu qu'on peut pointer du doigt. Elle peut protester contre l'abandon de Dieu et contre son absence, elle peut inciter à assumer la responsabilité de cette absence, et elle peut inviter à la reconnaissance de Dieu pour le transformer en un Dieu présent. La présence de Dieu ne peut être qu'une émotion intérieure. C'est une praxis (orthopraxie). Elle possède des critères dans la réalité même. La présence de Dieu réside dans l'absence de pauvres. La présence de Dieu est à l'œuvre; elle agit. Par conséquent, le contraire de la pauvreté, ce n'est pas l'abondance de choses, mais bien plutôt la plénitude de la vie qui se constitue à partir de la reconnaissance mutuelle entre des sujets corporels et soumis à des besoins. En ce sens, la théologie de la libération est une orthopraxie. Dieu ne dit pas ce qu'il faut faire. Sa volonté, c'est de libérer le pauvre, mais le chemin de la libération, il faut le chercher. De l'ana-

hébreu * kamokha * du début du verset, on peut lire le tout autrement: «Aime ton prochain; cetre œuvre est comme toi-même»; «aime ton prochain; c'est toi-même»; «c'est cet amour du prochain qui est toi-même». (De Dieu qui vient à l'idée. (Problèmes et controverses), Paris, J. Vrin, 1982, p. 144). 
lyse de la réalité dépend ce qui sera considéré comme la volonté de Dieu. Par conséquent, on ne peut jamais savoir la volonté de Dieu sinon en passant par une analyse de la réalité qui ne peut jamais faire abstraction des sciences sociales. Et les résultats des sciences sociales ont une incidence directe sur ce qui, pour l'orthopraxie de la théologie de la libération, est la volonté de Dieu.

La théologie de "l'orthodoxie" est différente. Elle se cantonne dans des affirmations dogmatiques sans les chercher dans un lieu concret et historique. Voilà pourquoi il est si facile pour elle de se ranger du côté de la domination. La domination est toujours abstraite; elle revendique une validité indépendamment des situations concrètes et historiques. Cela attire l'attention sur cette constatation que dans la dispute entre la théologie de la libération et "l'orthodoxie" théologique fermée, c'est à peine s'il existe des discussions sur le contenu dogmatique. Cette "orthodoxie" proclame le contenu dogmatique et la théologie de la libération le reconnaît aussi. En ce sens, il n'y a pas de conflit religieux. Cette situation est complètement différente des conflits religieux du Moyen Âge européen. À cette époque, les conflits tournaient autour des contenus de la dogmatique. Le schisme entre l'Église d'Occident et l'Église orientale éclata au sujet de la formule trinitaire, c'est-à-dire, à savoir si l'Esprit-Saint était engendré par Dieu le Père et par Dieu le Fils ou seulement par Dieu le Père. Dans le cas des Cathares, il s'agissait de la question de la résurrection des corps. Même chose lors de la Réforme où des conflits du même type prédominèrent, par exemple sur l'interprétation de l'Eucharistie et la signification du ciel des saints.

\section{2. Économie et théologie au début de la théologie de la libération}

Quand à la fin des années 70 surgit le conflit au sujet de la théologie de la libération, les raisons déclarées n'ont à peu près rien à voir avec des divergences d'ordre dogmatique. Par conséquent, la discussion ne concerne pas des contenus théologiques au sens formel, mais tourne plutôt autour de la signification concrète de ces contenus. Toutefois, étant donné que la théologie officielle, qui se proclame orthodoxe est exclusivement dogmatique, cette discussion oppose la position orthodoxe qui réduit le contenu théologique à l'énoncé de vérités éternelles vides, avec la position des théologiens de la libération qui défendent la concrétisation historique de cette même foi. Par conséquent, l'usage des sciences sociales dans la théologie de la libération en vient à jouer un rôle-clé dans ce conflit. 
Ce conflit se manifesta publiquement pour la première fois pendant la période du gouvernement de l'Unité Populaire, au Chili, entre 1970 et 1973. La théologie de la libération avait surgi dans les années précédentes, principalement durant la seconde moitié des années 60 . Elle n'est pas d'abord apparue dans les milieux académiques, mais plutôr à partir de l'activité pastorale des Églises. Il s'agissait surtout de prêtres et de pasteurs qui travaillaient dans des milieux populaires des pays latinoaméricains. Les premières publications furent avant tout des manuscrits miméographiés qu'on distribuait lors de rencontres ou encore par le courrier. $\ddot{A}$ la fin des années 60 , paraissent les premiers livres (Hugo Assmann, Gustavo Gutiérrez, José Míguez Bonino, Juan Luis Segundo). Les idées de ces auteurs se propagent et gagnent vite de l'influence dans les séminaires et les facultés de théologie. Elles créent un courant d'opinion en Amérique latine qui s'exprimera avec encore plus d'intensité au Chili après la victoire électorale de l'Unité Populaire en 1970.

Dès ses débuts, cette théologie de la libération qui surgit se trouve très liée aux mouvements populaires de la décennie des années 60 . Ces mouvements sont alors en plein essor. Au Chili, ils se cherchent une expression politique autant dans les partis de l'Unité Populaire que dans le parti démocrate-chrétien. Ce sont là des mouvements qui aspirent à une intégration économique et sociale des groupes populaires. Ce problème est de plus en plus palpable, surtout à partir de deux tendances qui se dessinent durant les années 60 . L'une d'elle touchait la siruation de marginalité qui se faisait sentir principalement dans les centres urbains avec leurs ceintures de misère mais aussi dans les régions rurales avec les paysans sans terre et ceux qui ne possédaient que des parcelles de terre (minifundistes). L'autre tendance, c'était la stagnation de l'emploi. Même si se poursuivait une expansion parfois très forte de la production industrielle, celle-ci était due principalement à des augmentations de la productivité du travail et, par conséquent, ne donnait pas lieu à une croissance du nombre d'emplois. La situation de marginalité elle-même en est ainsi venue à revêtir le caractère d'une exclusion structurelle et non d'un simple phénomène de transition.

C'est à partir de cette problématique qu'on peut expliquer le fait que les mouvements populaires aient mis l'emphase sur des changements de la structure économique et sociale elle-même. Durant les années 60 , plusieurs des mouvements populaires espéraient encore une possible solution à l'intérieur d'un capitalisme de réformes, comme le proposait la démocratie chrétienne chilienne. Toutefois, surtout après 1968, les mouvements populaires se tournèrent vers l'Unité Populaire. C'est alors qu'est survenue 
la division de la démocratie-chrétienne au Chili, une partie décidant d'accorder son appui à l'Unité Populaire.

Ces changements d'orientation politique des mouvements populaires correspondaient à une expérience profonde. On s'etait rendu compte, en effet, qu'il n'existait aucune possibilité de développement comportant une intégration économique et sociale dans le cadre des structures capitalistes existantes. On parla d'abord de la nécessité d'un développement néo-capitaliste, puis plutôt d'un développement socialiste. Au plan théorique, on analysait cette situation à partir de la théorie de la dépendance. La majorité des théologiens de la libération partageaient cette expérience et cette évaluation de l'incapacité des structures capitalistes, avec leur logique propre, de créer une société capable de solutionner les problèmes économiques et sociaux des groupes populaires. Ils avaient aussi tendance à croire qu'un développement intégral ne pouvait se réaliser que par un changement profond des structures capitalistes elles-mêmes. C'est pourquoi ils formèrent une organisation continentale qui prit le nom de "Chrétiens pour le socialisme " et qui tint sa première rencontre importante à Santiago du Chili, en mars 1972. Cette organisation était œecuménique car elle rassemblait des théologiens de la libération tant catholiques que protestants.

Cette critique du capitalisme et cette recherche d'une alternative visant à la transformation des structures capitalistes elles-mêmes mena la théologie de la libération naissante à un conflit autant avec la théologie officielle, comme au Chili par exemple, qu'avec l'Église carholique, et en particulier avec sa hiérarchie. Cette Église avait été une alliée étroite de la démocratie chrétienne dans les années 60 . Quand ce parti politique s'est orienté vers des positions anti-socialistes et anti-communistes, l'Église a pris la même direction.

Tourefois, il était bien difficile de réfuter l'expérience des théologiens de la libération et la théorie de la dépendance à partir de laquelle ils interprétaient cette expérience. Aujourd'hui, après trente années de poursuite et de renforcement du capitalisme en Amérique latine, nous pouvons constater que cette interprétation du capitalisme latino-américain de la part des théologiens de la libération - et des thèses de la théorie de la dépendance qu'ils ont alors appuyées - s'est totalement confirmée. Toutefois, il est actuellement plus difficile de concevoir des alternatives qu'on le croyait il y a trente ans. En effet, le capitalisme latino-américain, poussé à sa logique extrême, a encore aggravé la marginalisation de la population en une exclusion sans issue. 
Voilà pourquoi, dans les années 70 , même si la théologie officielle et l'Église catholique du Chili cherchaient à créer un conflit avec les tenants de la théologie de la libération, ils ne possédaient aucun argument. D'une part, il n'existait pas de différences majeures au niveau du contenu dogmatique de la foi et, d'autre part, la critique du capitalisme par les théologiens, tout au moins dans ses grandes lignes, n'était pas réfutable. Par conséquent, il n'y eut aucun débat, ni non plus d'argumentation rationnelle. On s'est simplement employé à dénoncer la nouvelle théologie.

\section{Dénonciation de la théologie de la libération}

Une théologie institutionnalisée, qui agit au nom d'un corpus dogmatique avec la prétention de posséder la vérité éternelle, ne peut s'actualiser dans l'histoire. Par conséquent, pour affronter la théologie de la libération, elle ne peut la démanteler, mais seulement déclarer inappropriée et même perverse la manière dont cette théologie se concrétise. Elle ne peut débattre de cette concrétisation, car, dans ce cas, elle devrait accepter que la théologie est et doit être une théologie concrète et historique.

La dénonciation devient la seule porte de sortie de cette orthodoxie obtue. $\mathrm{E}_{t}$ comme les théologiens de la libération recourent très souvent à des éléments de la théorie marxiste pour penser théoriquement leur expérience vécue, on dénonce donc la théologie de la libération comme marxiste. Dans une société comme la société bourgeoise moderne, Marx est, bien sûr - au sens orwellien - "la non-personne " que tout le monde doit attaquer à cor et à cri pour démontrer sa fidélité aux valeurs du monde qui s'est auto-proclamé "libre". Marx est au monde libre ce que Trotsky est au monde soviétique. C'est la "non-personne " dans laquelle est supposé s'être incarné le mal. Par conséquent, dénoncer le marxisme de la théologie de la libération implique une condamnation irrationnelle et idéologique de cette théologie, sans se donner la peine de réfuter ses préoccupations concrètes. Ce qui est concret se dissipe sans qu'on ait besoin d'en discuter. L'autre se révèle comme un ennemi, indépendamment de ce qu'il pense.

Afin que la dénonciation serve à cette fin, on transforme la pensée de Marx en une grande magie à laquelle on ne peut échapper. Celui qui s'en approche, se perd. C'est une grande turbulence comme le remous Maelström. Si quelqu'un s'en approche un tant soit peu, le remous va l'entraîner de façon irrémédiable, va l'avaler et le conduire à la perdition. Ce n'est plus une théorie mais la tentation du mal lui-même. Le cardinal Ratzinger résume très bien la vision de ce Lucifer : 
La pensée de Marx constitue une conception totalisante du monde dans laquelle de nombreuses données d'observation et d'analyse descriptive sont intégrées dans une structure philosophico-idéologique, qui commande la signification et l'importance relative qu'on leur reconnaît (...) Ainsi la dissociation des éléments hétérogènes qui composent cer amalgame épistémologiquement hybride devient impossible, de sorte qu'en croyant n'accepter que ce qui se présente comme une analyse, on est entraîné à accepter en même temps l'idéologie ${ }^{3}$.

Toutefois, les théologiens avaient uniquement affirmé que l'option pour les pauvres entrait en conflit avec la logique de la structure du capital. Par conséquent, si cette option était prise au sérieux et d'une manière réaliste, il fallait dépasser cette logique. Ce dépassement, ils l'appelèrent socialisme. La discussion aurait dû tourner autour du questionnement des théologiens de la libération pour savoir si, dans les faits, les choses se passaient ou non comme ils le soutenaient. Il n'y eut pas un seul mot sur cela. Les faits ne comptaient pas.

La raison du rejet ne tenait pas à ce que les théologiens aient utilisé des théories marxistes. De toute façon, la condamnation aurait été la même dans le cas où ils ne se seraient pas référés à Marx. Le seul fait de conclure que l'option pour le pauvre entrait en conflit avec la logique de la structure du capital "prouvait " hors de tout doute leur allégeance marxiste. La condamnation idéologique interdisait même la question; il était donc inutile de discuter de la validité d'une réponse.

Parallèlement à la dénonciation magique du marxisme, la dénonciation anti-utopique apparut déjà au temps de l'Unité Populaire au Chili. La dénonciation anti-utopique n'était que l'autre face de cet anti-marxisme magique. Encore une fois, on substituait une dénonciation à la discussion des situations concrètes et historiques. On ne vit pas non plus de débat sur ce qu'on entendair par utopie ni d'analyse de la problématique. Les théologiens de la libération demandaient des changements de structures afin que la société puisse trouver une solution au problème de la pauvreté. Par conséquent, ils ne demandaient pas la réalisation d'utopies. Ils visaient un objectif très réaliste, même s'ils savaient que le réalisme de ce projet dépassait les possibilités de la société capitaliste dans laquelle ils vivaient.

3 Libertatis nuntius, VII, 6. Voir à ce sujet Franz HINKELAMMERT, « Befreiung soziale Sünde und subjekrive Verantwortlichkeit », dans Hermann-Josef VENETZ, Herbert VORGRIMLER (dir.), Das Lehramt der Kirche und der Schrei des Armen. Freiburg-Münster, Edirions Exodus y Liberacion, 1985, pp. 60-76. 
Cela aurait, bien sûr, impliqué un débat concernant les dimensions utopiques des objectifs politiques et une critique correspondante des contenus utopiques relativement au réalisme du changement des structures. Ce sont les théologiens de la libération qui ont commencé cette critique. La dénonciation anti-utopique, au contraire, n'a fait que les dénoncer comme des suppôts de Satan, évitant tout dialogue 4 .

Cela conduisit à une situation où la condamnation la plus forte des "Chrétiens pour le socialisme " qu'ait faite l'Église catholique chilienne eut lieu après le coup d'État militaire, alors que ces derniers étaient déjà persécutés par le terrorisme d'État du système totalitaire de Sécurité nationale qui s'organisait au Chili à cette époque.

La condamnation formelle du regroupement des Chrétiens pour le socialisme fut toute une histoire. Cette condamnation fut décidée en secret et sans être publiée par la Conférence épiscopale chilienne en avril 1973. Deux jours après le coup d'État militaire, donc le 13 septembre 1973, la même Conférence approuva un document additionnel. Ces condamnations furent mises en circulation le 26 octobre 1973 et publiées définitivement en avril 1974. Au cours de cette période, plus de 60 prêtres furent expulsés du Chili et quelques-uns torturés. Plusieurs membres laïcs des Chrétiens pour le socialisme furent tués, torturés ou emprisonnés. Le conflit avait débouché sur la répression des Chrétiens pour le socialisme au moyen du terrorisme d'État ${ }^{5}$.

4 Comblin résume ainsi certe critique de l'uropie de la part des théologiens de la libération : "L'avenir a été disposé par Dieu et demeure toujours hors d'atteinte de l'homme : c'est l'homme renouvelé, l'homme de l'alliance nouvelle... L'avenir se vit en vivant le présent. On ne peut sacrifier le présent en faveur de l'avenir, au contraire l'avenir doit être vécu et réalisé dans le présent, sous forme d'image ou de ressemblance. Ne pas sacrifier l'homme présent en vue d'une fraternité et d'une paix future, mais vivre cetre paix future en une image présente, imparfaite mais valide et réelle. D'autre part, la signification du présent ne réside pas dans la satisfaction immédiate qu'il accorde, mais dans l'image de l'avenir qu'il permer de réaliser » (José COMBLIN, Mensaje, juillet, 1974, p. 298). Voir aussi Franz HINKELAMMERT, Ideologías del desarrollo y dialéctica de la historia. Buenos Aires, Editorial Universidad Católica de Chile-Paidós, 1970.

5 Le Cardinal de Santiago lui-même, Raúl Silva Henriquez, déclara, lors d'un voyage en Italie que les Chrétiens pour le socialisme avaient pris un chemin qui «les faisait renoncer concrètement à leur christianisme...» (Avvenire, cité par El Mercurio, 25 octobre 1973). 


\section{La théologie de la libération et les dictatures de la Sécurité nationale}

Ce n'est pas seulement pour les Chrétiens pour le socialisme mais aussi pour les théologiens de la libération en général que le coup d'État militaire du 11 septembre 1973 signifia une coupure profonde. Il ne s'agissait pas d'un coup d'État traditionnel par lequel un groupe militaire assume le gouvernement, assurant la continuité d'une société bourgeoise déjà en place. Le coup d'État militaire chilien fut un coup de la Sécurité nationale. Le gouvernement militaire a assumé la tâche de restructurer dans ses fondements mêmes la société bourgeoise chilienne, selon un schéma idéologique préconçu. Il s'agissait d'établir une société à partir de principes abstraits n'ayant aucune relation avec l'histoire du Chili, et qui n'existait pas non plus dans le capitalisme mondial réel d'alors qui était un capitalisme réformiste et interventionniste. C'était la première fois dans l'histoire contemporaine qu'apparaissait un régime strictement néolibéral. Celui-ci s'imposa en utilisant le terrorisme d'État, selon un modèle abstrait déduit des principes d'une totalisation du marché capitaliste. C'est à cela qu'est dû le caractère jacobin de ce coup d'État ${ }^{6}$.

La politique de la Junte militaire chilienne visait un changement de la société tout entière. Il ne s'agissait pas simplement d'éliminer toute trace de la politique de l'Unité Populaire, mais bien de transformer au plus profond le capitalisme qui avait prévalu jusque-là. Ce capitalisme en avait été un de réformes, de type interventionniste et qui avait donné lieu à une société civile bien développée au sein de laquelle les organisations populaires avaient joué un rôle légitime et important. Les idéologues de la Junte militaire virent dans ce capitalisme réformiste la source du surgissement de l'Unité Populaire. En effet, celle-ci n'avait pas fait autre chose que de conduire à son terme logique ce même réformisme, c'est-àdire à un dépassement des limites de la structure capitaliste elle-même. La politique de l'Unité Populaire avait poursuivi ce réformisme avec des conséquences qui mirent en échec la structure même du capitalisme.

6 L'analyse que Hegel fait du jacobisme, est absolument pertinente pour expliquer le coup d'État militaire chilien et sa politique postérieure : « Ces abstractions, développées jusqu'à se transformer en force, ont produit réellement d'un côté, le premier - et ce depuis les origines du genre humain - prodigieux spectacle de tout recommencer à neuf et par la pensée, la constitution d'un État réel, bâti sur les ruines de tout ce qui existait dans ce pays, et de vouloir lui donner comme fondement la prétendue rationalité. D'autre part, étant donné que ce sont seulement des abstractions privées d'idées, elles ont fait de cette tentative un événement trop terrible et trop cruel ». (HEGEL, Filosofía del derecho, par. 258). 
C'est pourquoi la Junte militaire adopta deux lignes d'action principales. D'un côté, elle se consacra à détruire à fond toutes les organisations populaires, et par-dessus tout les syndicats, les organisations d'entraide entre voisins et les coopératives. Comme ces organisations étaient socialement et politiquement très enracinées, cet objectif impliquait la destruction de tous les partis populaires. Le terrorisme d'État joua là son rôle principal. Le coup d'Érat militaire a à peine visé à consolider le gouvernement, ce qui fut vite assuré dès les premiers jours du coup. S'installa alors une politique de la terreur qui dura plus de dix ans et qui parvint d'une manière très efficace à éliminer tout pouvoir populaire quel qu'il soit. La politique de la Junte visa, par ailleurs, à changer l'État. L'État réformiste de l'Unité populaire et son interventionnisme dans le marché représentait l'autre face de la force des mouvements populaires. Il fut transformé en un État anti-réformiste et anti-interventionniste au service du tout marché. La privatisation des fonctions de l'État dans le champ économique et social, et par conséquent des entreprises publiques, constitua alors, en Amérique latine, le premier cas d'une application principialiste de schémas abstraits empruntés à l'École de Chicago par les protagonistes du processus. Il s'agissait de la politique qui allait être rapidement adoptée par le Fonds monétaire international (FMI), au niveau mondial, sous le nom d' « ajustement structurel ». Milton Friedman lui donnera, plus tard, le nom de "capitalisme total" ".

C'est dans ce nouvel environnement que les réflexions des théologiens de la libération en Amérique latine se sont inscrites. Même s'il n'y eut pas de rupture au sein de cette théologie, elle connut cependant des changements importants sous cerrains aspects. Alors qu'au cours de la période précédant le coup d'État chilien, le point de vue libérateur ou libérationniste avait prévalu, la perspective de la résistance prenait maintenent le pas. Sans être absolument nouvelle, cette perspective allait maintenant occuper le premier plan. Cela allait de pair avec le changement intervenu dans la société. Il y avait toujours eu un travail intense réalisé à partir des communautés ecclésiales de base et ce travail avait été inextricablement lié à celui des organisations populaires à tous leurs niveaux. Mais maintenant les organisations populaires étaient les plus persécutées et elles avaient perdu beaucoup de leur vigueur. Les communautés de base se mirent, par conséquent, à jouer un rôle beaucoup plus important. De plus, comme les Églises maintenaient certains espaces à l'abri de la répression,

7 Cité par Guy SORMAN, «Sauver le capiralisme : le dernier combar de Milron Friedman », Le Devoir, Montréal, 5 avril, 1994. 
en bien des endroits, les communautés sont devenues les seuls lieux où l'activité populaire restait possible. À la place des organisations populaires, apparurent beaucoup de groupes de défense des droits humains. Il n'y a qu'en Amérique centrale où on s'est trouvé dans une situation différente, spécialement au Nicaragua et au Salvador. Cette tendance se renversa cependant au cours des années 80 .

Au centre de la théologie de la libération apparait maintenant le thème de l'idolâtrie et du Dieu de la vie face aux dieux de la mort. Le thème de l'idolâtrie s'inscrit dans une longue tradition d'origine juive. D'après cette tradition, l'idole est un dieu dont l'expérience et la vénération conduisent à la mort. Il ne s'agit pas de la question de savoir si, en un certain sens ontologique, l'idole existe ou non, mais du fait que l'idole soit vue comme une force de mort et soit vénéré comme un dieu. L'idole est un dieu de mort et, par conséquent, elle entre en collision avec le Dieu de la vie. En corollaire, le Dieu de la vie est vu comme un Dieu dont l'expérience et la vénération produisent la vie et non la mort. Comme la théologie de la libération est une théologie faisant pleinement droit à la corporéité, de même la vie ou la mort apparaissent sous un jour où la vie corporelle est l'exigence ultime de toute vie. Si donc le corps ne vit que comme un corps animé, ou habité par une âme, l'âme ne vit pas sans le corps. (Gracia suponit naturam).

C'est pourquoi, pour les théologiens de la libération, le problème fondamental n'est pas celui du théisme et de l'athéisme mais bien plutôt celui du Dieu de la vie et de l'idolâtrie. La position contraire ce n'est pas l'athéisme. La foi en Dieu peut être ou pas idolâtrique, tout comme l'athéisme. Un athéisme dont l'expérience mène à la mort est idolâtre, mais un athéisme dont l'expérience conduit à la vie ne l'est pas. La vie et la mort, voilà les critères, et non pas une abstraction métaphysique. Dans le peuple de Dieu aussi il y a des athées. Bien sûr, l'affirmation de la vie reste toujours vue à partir de la reconnaissance mutuelle entre des sujets qui se reconnaissent comme des être naturels et soumis à divers besoins. La vie et la mort en tant que critères, on les retrouve de nouveau comme critères de l'option pour le pauvre. Néanmoins, le pauvre possède maintenant une nouvelle dimension. Il n'est plus uniquement pauvre, il est en même temps une victime. Il l'est en tant que pauvre et en tant que persécuté par le système de répression de l'État.

À partir de cette analyse de l'idolâtrie et de sa victime, la théologie de la libération analyse toujours davantage les processus de victimisation. La théologie officielle est maintenant considérée comme une théologie du sacrifice, une théologie du Dieu qui exige des sacrifices. La théologie de 
la libération développe une forte critique de cette sacrificialité théologique et réalise certe critique à partir de l'analyse de la sacrificialité du système économique et social imposé en Amérique latine. On découvre toute une histoire de la sacrificialité inhérente à la conquête même de l'Amérique, et des réactions de la première heure en appui aux indigènes. Gustavo Gutiérrez revient à la discussion sur la théologie de la conquête et récupère la figure de Bartolomé de las Casas comme un ancêtre-clé de la théologie de la libération ${ }^{8}$.

D'autre part, on se met à approfondir la problématique de la relation entre l'économie et la théologie, spécialement au $\mathrm{DEI}^{9}$. Cette recherche se centre aussi sur le thème du sacrifice comme un élément constitutif du système économique et social actuel ${ }^{10}$. On découvre la sacrificialité de la culture occidentale elle-même et on renoue avec la tradition judéochrétienne de l'anti-sacrificialité.

\section{Le conflit autour de la théologie de la libération}

Il y a toujours eu un conflit latent entre la théologie de la libération, d'une part, et la théologie officielle et les Églises qui endossent cette dernière, d'autre part. Nous avons vu, déjà, comment ce conflit devint ouvert vers la fin du gouvernement de l'Unité Populaire et au cours de la première année de la Junte militaire chilienne. Mais déjà, avec le Rapport Rockefeller, à la fin des années 60 , un autre conflit avait surgi. Je veux parler du conflit avec le pouvoir politique et l'Empire. Pour l'Empire, la théologie de la libération constitue, à plusieurs titres, un péril. Une première raison importante pourrait être dite idéologique et a joué un rôle en particulier pendant la Guerre froide. Cet affrontement, interprété de façon manichéenne, avait besoin de camps très clairement délimités.

La théologie de la vicrime possède aussi un enracinement dans la théologie allemande au temps du nazisme. Voir Gustavo GUTTIÉRREZ, « Los límites de la teología moderna : un texto de Bonhoeffer », dans ID., La fuerza histórica de los pobres. Lima, CEP, 1979; Franz J. HINKELAMMERT, « La critica de la religion en nombre del cristianismo: Dietrich Bonhoeffer", dans XXX, Teología alemana y teologia latinoamericana de la liberación. Un esfuerzo de diálogo. San José, DEI, 1990, pp. 4565.

9 Hugo AssmanN, Franz Hinkelammert, L'idolatrie du marché. Critique théologique de l'économie de marché. (Libération), Paris, Cerf, 1993, 375 p.

10 Franz J. HinkelammerT, « Paradigmas y metamorfosis del sacrificio de vidas humanas », dans H. ASSMANN (dir.), Sobre idolos y sacrificios. René Giratd con teologos de la liberacion. San José, DEI, 1991, p. 157-179. 
L'Empire se définissait lui-même comme Occident chrétien, comme le Royaume de Dieu face au royaume du mal athée. L'Empire occidental semblait, comme l'archange Michel, lutter du côté de Dieu contre un empire qui s'opposerait à Lui. Même si le fondement de la légitimité de la société bourgeoise n'est pas chrétien, mais repose sur des mythes séculaires, cette légitimité a un besoin essentiel de la dimension religieuse pour pouvoir se raccorder à la transcendance. Croire en Dieu et lutter aux côtés du capitalisme contre les ennemis de celui-ci semblaient coïncider. Cette identification est encore plus forte aux États-Unis qu'en Europe, même si on la trouve là aussi. Cela mène à ce qu'on appelle, aux ÉtatsUnis, la religion civique (civil religion) qui est une religiosité sous-jacente à l'american way of life elle-même. C'est cette religiosité qui englobe toutes les religions particulières. Par conséquent, la tolérance religieuse face aux diverses confessions exige de leur part le respect du cadre fixé par certe religion civique. La religion est considérée comme une affaire privée, pour autant qu'elle s'inscrit dans la religion civique reconnue comme la religiosité publique.

La théologie de la libération menaçait cette homogénéité religieuse (et chrétienne) de l'Empire. Quand se pratique le discernement des dieux, quelques-uns d'entre eux ont bien raison de s'inquiéter. La question était d'autant plus délicate que les thèses de la théologie de la libération recevaient un accueil positif de la part de plusieurs Églises des États-Unis et de l'Europe, ainsi que du public en général. La condamnation manichéenne des mouvements populaires, de leurs protestations et de leurs revendications pour des changements de structures, était rendue plus difficile par le fait que des courants importants de ces mouvements s'inspiraient de leur foi religieuse. De plus, le public en général pouvait douter du simplisme des idéologies de la Guerre froide.

Quelque chose de semblable, mais sous un autre rapport, se produisit dans les mouvements populaires en Amérique latine. La théologie de la libération était un des courants de pensée qui permettait de se sortir de l'étroitesse de l'orthodoxie marxiste, surtout si l'on tient compte de la façon dont cette dernière était diffusée par la littérature publiée par l'Académie des sciences de Moscou. On se lassait rapidement de cette orthodoxie parce qu'elle ne réussissait pas à penser la réalité que les membres de ces mouvements vivaient. Elle était aussi abstraite et loin de cette réalité que pouvaient l'être les idéologies du marché. Dans les années 70, l'ennui que dégageait cette orthodoxie marxiste était notoire. C'est alors qu'on découvrit une pensée de Marx irréductible à cette orthodoxie. Plusieurs courants nouveaux de la pensée marxiste apparurent. Quoi qu'il en soit, pour ces mouvements populaires, l'apparition d'une 
théologie qui pensait le monde d'un point de vue qui leur était proche et qui permettait de vivre la foi en participant dans les luttes de libération constitua un apport important. Même si la majorité des théologiens n'étaient pas anti-marxistes, et même s'ils s'inspirèrent de la pensée marxiste pour l'analyse de leur réalité, ils ont toujours maintenu une position critique face au marxisme, ce qui renforça de manière effective les mouvements populaires.

Tout cela était bien suffisant pour que l'Empire réagisse. Le Rapport Rockefeller donna le signal dès la fin des années 60. L'Empire commença alors à développer sa propre théologie, basée d'abord sur le rejet de la théologie de la libération et, plus tard, sur la récupération de celle-ci.

Au cours des années 70 apparurent des centres théologiques d'un caractère absolument nouveau. Le premier fut le Département de théologie de l'American Enterprise Institute, dirigé par Michel Novak. Sa raison d'être était la lutte contre la théologie de la libération en Amérique latine et contre ses répercussions aux États-Unis. Peu de temps après, on fonda l'Institut pour la religion et la démocratie, sous la direction de Peter Berger, un organisme qui a le même but que le précédent, mais qui agit plutôt au niveau de l'État, des organisations politiques et des Églises des États-Unis. Les ouvrages de Michel Novak, qui ont été publiés en espagnol, ont été distribués par les organisations patronales d'Amérique latine et par les ambassades des États-Unis à la grandeur du continent. De plus, Novak fit des voyages en Amérique latine pour donner des conférences et participer à des tables rondes, rencontres organisées par les ambassades des États-Unis ou par les cercles patronaux locaux. Les organisations de patrons européens ont suivi l'exemple de l'American Enterprise Institute et ont mis sur pied leurs propres centres théologiques. Le Pentagone a aussi formé des spécialistes en ce domaine pour agir au sein des organisations militaires panaméricaines et au sein des services secrets. Et quand un jour, un théologien de la libération fut torturé, le tortionnaire était l'un de ces spécialistes. Depuis la fin des années 80 , le FMI a développé sa propre réflexion théologique et le Directeur général lui-même du FMI, Michel Camdessus, est entré publiquement dans le jeu. Les principaux journaux se sont faits les porte-parole de la nouvelle théologie de l'Empire, une théologie dite "de la libération", qui est maintenant développée et publicisée. Le document de Santa Fe de 1980, qui a pensé et formulé la plate-forme électorale de la première présidence de Ronald Reagan, a désigné le front formé par l'Église populaire et la théologie de la libération en Amérique latine comme une des principales préoccupations de la Sécurité nationale des États-Unis. 
Jusqu'au milieu des années 80 , les arguments utilisés contre la théologie de la libération étaient semblables à ceux utilisés par la théologie officielle. On attaquait surtout l'analyse marxiste qui était présentée comme un élément théorique essentiel dans l'élaboration de la théologie de la libération et de ses formulations utopiques d'un avenir libéré. Une différence notable distinguait cependant cette approche : l'anti-utopisme de cette théologie de l'Empire y était plus radical que celui de l'orthodoxie théologique et que celui des Églises opposées à la théologie de la libération.

L'officialisme théologique reproche surtout aux théologiens de la libération d'avoir une fausse utopie. Mais elle ne leur reproche pas comme tel de promouvoir une utopie. Comme théologie chrétienne, elle maintient sa propre vision du Royaume de Dieu qui viendra, et des cieux où on espère parvenir. On ne peut reprocher à la théologie de la libération son espérance du Royaume de Dieu. On lui reproche cependant d'interpréter ce Royaume en des termes matériels, corporels et terrestres, prétendant par là qu'il s'agit d'une fausse conception du Royaume. Le Royaume de Dieu de l'orthodoxie institutionnalisée, au contraire, est conçu comme un royaume de purs esprits pour lesquels leur corporéité reste quelque chose d'éthéré et surtout d'éphémère. Par ailleurs, en effet, le Royaume de Dieu imaginé par les théologiens de la libération est une Terre nouvelle, c'est "cette terre sans la mort ", c'est un royaume où les nécessités corporelles pourront trouver à être satisfaites. L'orthodoxie voit cela comme quelque chose de "matérialiste", c'est-à-dire comme une fausse espérance à laquelle elle oppose sa vision de l'espérance "véritable 11 ». Néanmoins, on peut dire qu'elle ne nie pas la vision d'un Royaume de Dieu à venir.

La théologie de l'Empire des années 70 et celle de la première moitié des années 80 est différente. Elle est nettement anti-utopique. Elle oppose un monde sans espérance à la vision utopique de l'espérance. Même si elle continue d'absolutiser le marché et de présenter d'une manière utopique sa main invisible (le marché comme lieu de la

11 "La doctrine marxiste de la fin des temps est une promesse d'un salut intramondain. Karl Marx a sécularisé le destin du peuple juif - l'esclavage en Égypte et l'exode vers la terre promise - et l'espérance du salut messianique de l'Ancien Testament pour les transposer dans notre temps, le temps après Jésus-Christ, une réduction perturbatrice et une simple « singerie » [Nachäffung : agir comme un singe] du salut qui, en Jésus-Christ, a été donné à toute l'humanité. Le marxisme est un antiÉvangile». (Josef HOFFNER, Christliche Gesellschaftslehre. Kevelaer, 1975, p. 171-172) 
"Providence») et la tendance de celui-ci à l'équilibre, elle n'établit pas de relations entre l'utopie du marché et le Royaume de Dieu. Par conséquent, la solidarité elle-même apparaît comme une perversion humaine et un atavisme ${ }^{12}$.

Il s'agit d'une théologie qui correspond au manichéisme de la Guerre froide. L'Empire se définit dans le cadre d'une lutte entre Dieu et le démon, et voit l'utopie comme l'incarnation du Royaume du mal représenté par le démon, alors qu'il se voit lui-même comme un règne du réalisme qui n'a pas besoin d'utopie. À la confrontation Dieu-Démon (Royaume du Mal) correspond la confrontation réalisme-utopie. Ce manichéisme absolu, $\mathrm{K}$. Popper l'a interprété dans le sens suivant : qui veut le ciel produit l'enfer.

Bien sûr, cette vision de l'utopie a créé des problèmes au sein même de la coalition conservatrice qui s'est formée dans les années 80 . Et nous avons vu, déjà, que sous sa forme pure, elle n'était pas acceptable pour "l'orthodoxie » théologique. Elle n'a pas non plus réussi à cimenter cette coalition politique avec le fondamentalisme chrétien américain, un des piliers du pouvoir du gouvernement Reagan. Ce fondamentalisme est, au contraire, hautement utopique et messianique, et comporte une vision nettement apocalyptique de l'histoire ${ }^{13}$. Et du fait même de son existence

12

«... les sociétés traditionnelle et socialiste offrent une vision monolithique. Elles insufflent dans toures les activirés une symbolique de solidarité. Le cœur humain a faim d'une telle nourriture. Des souvenirs ataviques hantent tous les gens libres. La " friche » centrale du capitalisme démocratique est semblable à un champ de bataille, où errent des individus isolés, dans une certaine confusion avec bien des accidentés. Néanmoins, à l'instar de l'obscurité totale de l'âme dans le voyage intérieur des mystiques, ce désert a une fonction de première nécessité... Le domaine de la transcendance, bien sûr, s'aborde à travers littérature et religion, famille et compagnons de route. Mais, en définitive, sa source est dans l'intériorité silencieuse de chaque être.» (Michael NoVAK, Une éthique économique. Les valeurs de l'économie de marché. Paris, Cerf/Institut La Boétie, 1987, p. 59-60). L'auteur conclut en citant Reinhold NIEBUHR : * les "fils de lumière" sont à bien des égards un plus grand danger pour la Foi fondée sur la Révélation que les "fils des ténèbres" (ibid., p. 75).

13 Il suffit, pour s'en convaincre, de considérer un livre comme J. Dwight PENTECOST, Eventos del porvenir. Estudios de escatología bíblica. Miami, Editorial Vida, 1984; ou encore: Hal LINDSEY, La agonia del gran planeta Tierra. Miami, Editorial Vida, 1988 (The Late Great Planet Earth. Grand Rapids (MI), Zondervan Publishing House, 1970). Ce dernier livre s'est vendu à plus de 15 millions d'exemplaires durant les années 70 aux États-Unis et fut le best-seller de la décennie. Lindsey n'en a pas moins été l'un des Raspoutine de la cour de Reagan. 
comme allié du gouvernement Reagan, ce fondamentalisme démentait quotidiennement l'idéologie de la Guerre froide avec la théologie de l'Empire basée sur cette idéologie. Simultanément à l'effritement visible des pays socialistes, s'est produit une "utopisation " grandissante de l'Empire lui-même. La politique de l'ajustement structurel, avec ses effets désastreux sur les pays du Tiers-Monde, avait besoin de promesses d'un avenir meilleur pour pouvoir se légitimer. Les " enfers " produits sur la terre exigeaient la promesse de cieux à venir. Le néo-libéralisme s'est donc luimême transformé en religion, avec ses conversions et son évangile du marché.

\section{La tentative de récupération de la théologie de la libération par la théologie de l'Empire}

À partir de tout cela se produisit une transformation de la théologie de l'Empire. De la dénonciation de la théologie de la libération, elle passa à sa récupération. Au milieu des années 80 , cette récupération bartait son plein, même si on avait pu noter sa présence en Amérique latine depuis le coup d'État militaire chilien ${ }^{14}$. Quand, en 1985, David Stockman, qui était issu d'une tradition fondamentaliste, renonce à son poste de responsable du budget du gouvernement Reagan, il publie un livre intitulé Le triomphe de la politique. Dans ce volume, il reproche à Reagan d'avoir trahi le modèle pur du néo-libéralisme en faveur du populisme et développe toute une théologie de la position néo-libérale qui fait rapidement école. Ce livre ne dénonce pas du tout les utopies, mais présente le néo-libéralisme comme l'unique manière efficace et réaliste de les concrétiser. Il attaque les «utopies" du socialisme pour les récupérer en faveur du prétendu réalisme néo-libéral. Selon Stockman, la menace ne vient pas de l'utopie comme telle mais bien de la fausse utopie, à laquelle il oppose son utopie «réaliste» du néo-libéralisme ${ }^{15}$.

14 La « Déclaration de principes » du gouvernement militaire chilien de 1974 allait déjà dans ce sens.

15 Stockman se présente comme un homme de gauche converti, qui aurait un temps été partisan d'une fausse utopie, mais qui aurait maintenant découvert la véritable utopie : « en un sens plus profond, bien sûr, la nouvelle doctrine de l'offre (il parle même de l'“évangile de l'offre") n'était rien d'autre qu'une réédition de mon vieil idéalisme social sous une forme nouvelle et, du moins le croyais-je, plus mûre. Le monde pouvait encore recommencer à neuf. Les crises économiques et sociales, qui devenaient plus aiguës, pouvaient être résolues. Les maux héréditaires anciens, tels le racisme et la pauvreté, pouvaient être réglés par des réformes profondes relevant des instances politiques. Mais par-dessus tout, la doctrine de l'offre représentait une 
Le Directeur-général du FMI lui-même, Michel Camdessus, s'est fait l'écho de cette théologie transformée de l'Empire. Il l'organise directement à partir de quelques thèses-clés de la théologie de la libération. Lors d'une conférence donnée à Lille, le 27 mars 1992, il s'est adressé aux patrons et dirigeants chrétiens de France, réunis pour leurs assises nationales ${ }^{16}$. Le milieu de sa conférence résume ses thèses théologiques les plus importantes. J'en citerai de larges extraits.

Certes le Royaume c'est ce lieu : ces Cieux nouveaux et cette terre nouvelle où nous sommes appelés à entrer un jour; promesse sublime, mais le Royaume c'est en quelque sorte de la géographie, le Règne c'est de l'Histoire, une histoire dont nous sommes les acteurs, qui est en marche et qui est proche depuis que Jésus est venu dans l'histoire humaine. Le Règne c'est ce qui se passe quand Dieu est Roi et que nous le reconnaissons, et que nous faisons que le Règne s'étende comme tache d'huile, imprègne et renouvelle, unifie les réalités humaines. "Que ton Règne vienne... 17 "

Il oppose ensuite le pouvoir de ce monde et celui du royaume de Dieu :

L'un se fonde sur le pouvoir, l'autre sur le service; l'un, appuyé sur la force, va à la possession et à l'accaparement, l'autre au partage; l'un exalte le prince et ses barons, l'autre, l'exclu et le faible; l'un trace

alternative idéaliste au cynisme et au pessimisme actuels. $\gg$ Selon des extraits de ce livre parus dans Der Spigel $(1986, \mathrm{n} .16 \mathrm{ss}$.), les " réformes profondes relevant des instances politiques » désignaient, de fait, celles d'un ajustement structurel radical : " cela signifiait aussi la coupure subite de l'aide sociale aux nécessiteux aptes au travail... ce que seul un président de fer aurait pu imposer : "un tueur de monstres" ». Selon Stockman, Reagan a échoué parce qu'il n'a pas été capable de se transformer en « président de fer ».

16 Michel CAMDESSUS, "Marché-Royaume. La double appartenance », Documents Episcopat. Bulletin du secrétariat de la conférence des évêques de France, 12, juillet-août 1992. Camdessus a donné une conférence semblable à des entrepreneurs chrétiens du Mexique. L'Union internationale chrétienne des dirigeants d'entreprises (UNIAPAC) a tenu sa XIX ${ }^{\mathrm{e}}$ rencontre à Monterrey, du 27 au 29 septembre 1993, où Camdessus a développé le thème : "Le marché et le royaume face à la globalisation de l'économie mondiale ». Il y avair deux conférenciers à cette réunion. Le second était le Cardinal Echegaray. Voir : SELAT (Servicios Latinoamenicanos), Lima, Pérou, 17, 17 septembre 1993.

Ibid., p.4. 
des frontières, l'autre accueille; l'un s'appuie sur le spectaculaire et le médiatique, l'autre préferre la germination discrète du grain de sénevé. À l'opposé! et au cœur de ces différences, il y a celle-ci qui les récapitule toutes : le Roi s'identifie au PAUVRE... dans ce Royaume, qui y est juge, qui y est Roi? Dans l'Évangile la réponse nous est donnée de façon formidablement solennelle dans l'annonce et la perspective du Jugement dernier : - aujourd'hui - mon juge et mon roi, c'est mon Frère qui a faim, qui a soif, qui est étranger, nu, malade ou prisonnier... 18

Camdessus oppose, d'une part, le pouvoir, la possession et l'accaparement, le prince et les barons, les frontières, le spectaculaire et le médiatique et, d'autre part, le service, le partage, l'exclu et le faible, l'accueil et la discrète germination du grain de sénevé. Il oppose l'orgueil et l'humilité. Et il découvre que le FMI, l'ajustement structurel et toute la conception néo-libérale de la société incarnent justement cette humilité face à l'orgueil de ceux qui exercent une résistance. Il en arrive à la conclusion suivante :

Notre mandat? Il a retenti dans la synagogue de Nazareth, et l'Esprit nous est donné pour recevoir ce que les compatriotes de Jésus refusaient d'avaler, précisément la réalisation de la promesse faite à Isaïe $(61,1-3)$ à partir de notre histoire présente! C'est un texte d'Isaie que Jésus déroula et dit ( $\operatorname{Lc} 4,16-23)$ :

"L'Esprit du Seigneur est sur moi parce qu'il m'a conféré l'onction pour annoncer la bonne nouvelle aux Pauvres, proclamer aux captifs la libération, aux aveugles le retour à la vue, renvoyer les opprimés en liberté, proclamer une année de bienfaits accordés par le Seigneur. " Et Jésus n'a fait qu'une phrase de commentaire : "Aujourd'hui, cette écriture est accomplie pour vous qui l'entendez. "

Cet aujourd'hui là, c'est notre aujourd'hui et nous sommes, nous qui sommes en charge de l'économie, des administrateurs d'une partie en tout cas - de ces bienfaits de Dieu : le soulagement de la peine de nos frères et l'élargissement de leur liberté. Nous sommes ceux qui ont reçu cette Parole. Elle peut tout changer. Nous savons que Dieu est à l'œuvre avec nous pour faire grandir la fraternité ${ }^{19}$.

Ibid.

19 Ibid., p. 5. 
Le texte du Directeur général du FMI pourrait tout aussi bien avoir été écrit par un théologien de la libération. 11 formule ce que les théologiens de la libération considèrent comme le centre de leur interprétation du message évangélique, spécialement en ce qui concerne la promesse du Royaume de Dieu et l'option pour le pauvre.

Le texte cité n'est, toutefois, qu'une partie de la conférence. Ce qui précède et ce qui suit donnent au texte théologique un sens tout à fait opposé à la teneur qu'aurait eu un texte analogue dans une analyse faite dans la perspective de la théologie de la libération. Il s'élève là contre les «populismes». Ce mot, dans le langage du FMI, résume toutes les attitudes et politiques possibles qui n'endosseraient pas les strictes positions de l'ajustement structurel du FMI. Il s'érige avec virulence contre

...toutes ces formes de la démagogie populiste d'ores et déjà à l'œuvre et dont nous savons où elles mènent : à l'hyperinflation et à travers elle - avant même que le marché n'ait tenu ses promesses - à la débandade économique, à la montée de la misère et au retour des régimes dit "forts", disons plutôt à la fin des libertés 20 .

Camdessus transforme de cette manière l'option pour les pauvres en option pour le FMI. Quiconque veut plus, ou autre chose, que ce qui est accordé ou imposé par la politique d'ajustement structurel du FMI, provoquerait la «débandade économique, la montée de la misère et le retour de régimes dit "forts " ", c'est-à-dire "la fin des libertés". Cela nuirait au pauvre. Par conséquent, qui est avec le pauvre doit être, par la force des choses, en faveur du Fonds. Il n'y a pas d'alternative.

Comme Camdessus s'adresse à un public en majorité catholique, il interpelle sur le même ton la doctrine sociale catholique traditionnelle :

Bien sûr, le marché est le mode d'organisation économique le plus efficace pour accroître la richesse individuelle et collective; nous ne devons plus avoir à son égard cette attitude de ralliement embarrassé de quelques générations de nos frères catholiques sociaux qui disaient : cette sorte de "oui... mais". La cause est entendue et le Saint Père a bien clarifié le sujet dans «Centesimus annus». Le mar-

Ibid., p. 2. Dans une conférence donnée par Camdessus aux assises des Semaines sociales de France, en 1991, il a également opposé l'option pour les pauvres à ce qu'il appelle le populisme : «Prenons garde dans nos jugements à ne pas confondre option préférentielle pour les pauvres avec le populisme.» (Michel CAMDESSUS, « Libéralisme et solidarité à l'échelle mondiale ", dans XXX, Concurrence et solidarité. L'économie de marché, jusqu' où? Paris, ESF éditeur, 1992, p. 100. 
ché, par l'efficacité qu'il assure, peut permettre une solidarité plus forte. Par là, marché et solidarité ne s'opposent pas mais peuvent se rejoindre. De plus, l'économie d'entreprise, vous le savez bien, est une économie de responsabilité où l'homme peut donner toute sa dimension ${ }^{21}$.

En dehors du marché, il ne subsiste aucune autre activité possible, y compris la solidarité qui doit s'exercer par et dans les limites de sa logique. Camdessus présentera donc le FMI comme le grand organisme mondial dont la responsabilité est l'exercice de la solidarité. À cette fin, il raye cent années de doctrine sociale de l'Église catholique sans provoquer le moindre écho.

Camdessus se présente, bien sûr, comme un réaliste. Il parle de la relation entre le marché et le Royaume de Dieu, mais il essaie de les distinguer. On doit savoir "que le marché... n'est pas le Royaume ". Il voit très bien que le marché renferme une logique destructrice et autodestructrice :

Dès lors, si le marché est totalement laissé à ses mécanismes, il y a grand danger - il n'est pas nécessaire de remonter au XIX ${ }^{\mathrm{e}}$ siècle pour le voir - que les plus faibles soient piétinés. Dans sa logique pure, la mise à prix peut être mise à mort. " 30 deniers, marché conclu ". Ceci n'est pas un épisode singulier de l'histoire d'un prophète de Judée, c'est un élément permanent de l'histoire humaine. À partir de cette indifférence du marché à l'égard de la personne, vous pouvez rapidement retrouver l'origine profonde de bien des maux des sociétés avancées: pollution, accidents du travail, destruction des familles, exclusion et chômage, corruption, inégalités, etc.

Voilà pourquoi nous savons depuis longtemps que le marché doit être surveillé, encadré pour rester libre, mais aussi pour être juste. Voilà pourquoi on ne peut accepter la substitution, au fondamentalisme marxiste, d'un fondamentalisme de marché. Le marché ne peut être laissé à sa seule logique, car l'économie n'est pas de la technique, c'est de l'humain 22 .

Face à la destructivité du marché, il cherche maintenant à activer l'espérance du Royaume de Dieu : "Marché-Royaume, nous savons bien que ces deux là c'est à nous de les marier. En tout cas, dans nos vies...23 "Sous

Ibid., p. 3.

Ibid. 
le titre "l'espérance du Royaume » il dit : «Oui, cette réalité du marché si lourde de forces de mort et de vie. Cette réalité sur laquelle chacun d'entre nous, d'une façon ou d'une autre, a une prise, une responsabilité ${ }^{24}$.»

Toutefois, cette responsabilité dont parle Camdessus n'est rien d'autre que la responsabilité d'assurer le fonctionnement du marché. La logique du marché peut détruire le marché lui-même. Camdessus est bien conscient de ce problème. Le capitalisme des années 80 a été un capitalisme autodestructeur. Il détruit non seulement l'être humain et la nature, mais également sa propre possibilité de fonctionner. La corruption a cessé d'être un problème propre à l'Étar. Elle pénètre aujourd'hui les relations marchandes elles-mêmes et tend à les bloquer. De nos jours, le foyer principal de la corruption, ce n'est plus l'État mais bien l'entreprise capitaliste elle-même dans ses relations avec les autres entreprises capitalistes. Camdessus a besoin d'une éthique du marché, parce que cette éthique peut être pervertie par la logique propre au marché. Cette éthique, il la cherche dans la référence au Royaume de Dieu et dans le mariage entre le marché et le Royaume :

Il y a dans la vie du marché des pratiques auxquelles non seulement notre citoyenneté du royaume, mais aussi notre bonne citoyenneté de ce monde tout court, nous oblige à dire non. Et nous savons tous que ce non n'est pas facile, qu'il y faut souvent du courage et plus que du courage. Vous connaissez mieux que moi cette face d'ombre de la vie économique. J'ai compris que vous alliez en affiner le constat au sein de votre premier "module": "Qu'est-ce qui, autour de nous, concrètement, diminue, use, détruit: duretés, injustices, exclusions, manipulations des clients et du personnel... idolatrie de l'argent, vie de fou, etc. » Je ne m'y arrête pas davantage. La vie économique n'est pas que cela et il y a un vaste domaine où les deux surfaces, oui, d'une certaine façon se recouvrent. Laissez-moi m'y arrêter plus longtemps. C'est tout le domaine où le porteur de valeur du royaume non seulement n'impose pas je ne sais quel frein au dynamisme du marché mais lui apporte le "plus " qui manque au marché pour mieux servir l'homme intégral. C'est, en d'autres termes, tout le domaine où rationalité économique et construction du royaume convergent. Et il est vaste ${ }^{25}$.

Ibid., p. 4.

Ibid., p. 5-6. 
Mais qu'arrive-t-il lorsque cette rationalité et celle du Royaume ne convergent pas? Dans ce cas, selon Camdessus, il n'y a rien à faire. Le réalisme apparent débouche sur le même fondamentalisme qu'il prétendait critiquer. Si bien que, pour Camdessus, si le marché n'est pas le Royaume, il n'en continue pas moins d'en être l'unique présence possible. Le marché, avec sa logique, se transforme en limite eschatologique de toute l'histoire humaine. Surveiller le marché, pour Camdessus, ne signifie rien d'autre que de le rendre viable. Il souhaite intervenir dans le marché pour que celui-ci continue de fonctionner. Toutefois, le critère d'intervention n'est rien d'autre que d'assurer le fonctionnement du marché selon sa propre logique conçue comme la trace de Dieu dans l'histoire. Le marché n'est pas parfait, mais toute perfection qui ne serait pas issue de ce marché n'appartiendrait déjà plus à la praxis humaine. Le Royaume, c'est l'huile dont a besoin la machine du marché.

Selon Camdessus, il y a bien un royaume définitif au-delà du marché. C'est cependant un royaume qui est au-delà de l'histoire, qui ne se mêle pas des affaires du marché. C'est un Royaume eschatologique. C'est pourquoi Camdessus arrive à la conclusion suivante :

Le citoyen du Royaume - appelons-nous comme cela - est à la pointe de l'effort pour que reculent toutes les formes de peurs, les méfiances, l'accaparement «cette idolâtrie " comme dit saint Paul (Eph 5,5), et finalement pour que s'élargisse le champ du partage, celui où le Royaume déjà imprègne les réalités humaines, et que l'homme trouve un peu plus d'espace, de gratuité et d'épanouissement. Cela, en sachant très bien qu' i il y aura toujours des pauvres parmi nous ». Ce qui veur dire, entre autres choses - et il a dû en coûter cher à Jésus de dire cela - que le Royaume ne sera pas sur cette terre en tout cas jusqu'au jour où "il fera toutes choses nouvelles".

Cette tâche d'imprégnation des réalités humaines, nous ne la mènerons pas à bien sans que nos cœurs et nos intelligences soient élargis, renouvellés, "emplis de la Grâce d'en haut ". Pour qui exerce nos types de métiers, dans cette urgence au service des hommes, il n'y a pas d'autre solution j'en suis sûr et j'en suis loin - que la sainteté ou si vous voulez, de "revêtir » l'Homme Nouveau : cet homme pétri de terre mais qui - je reviens à saint Paul - "puisque le Christ est venu $d u$ Ciel, comme lui appartient au Ciel". Pétri de terre mais appartenant au ciel : la clé est là, et dans la Prière pour recevoir ce don ${ }^{26}$.

Ibid. , p. 8. 
Voilà une déclaration de l'Empire total, sans aucune issue ni sur la terre ni dans les cieux. La politique du FMI a été transformée en volonté de Dieu sur cette terre. Ce n'est plus une volonté exprimée sur un quelconque Sinaï, mais bien par la réalité elle-même. La réalité est telle que, si l'action humaine sort du cadre de la politique de l'ajustement structurel, les résultats seront nécessairement pires que la situation qu'on voulait changer. Il n'y a pas d'alternative parce que le simple fait d'en chercher une entraînerait, de manière infaillible, la détérioration de la situation. Voilà pourquoi opter pour le pauvre, c'est opter pour le réalisme. Et le réalisme impose de ne pas se préoccuper du pauvre. Ce que fait le marché ne souffre pas de remise en question. Le capitalisme s'est transformé en "capitalisme total ", comme l'appelle Friedman. L'option préférentielle pour le pauvre et l'option préférentielle pour le FMI sont devenues la même chose ${ }^{27}$. Il s'agit d'une position qui est maintenant très courante et qu'on retrouve dans un nombre incalculable de publications qui se font passer pour de la théologie de la libération 28 .

Cela représente, évidemment, une grande tentation pour les Églises. Dans la vision ouverte par Camdessus, on peut opter pour le pauvre sans entrer aucunement en conflit avec le pouvoir. La grande harmonie semble être advenue. Et c'est la main invisible du marché qui l'aurait apportée. Ainsi la théologie de la libération semble maintenant faire partie de "l'orthodoxie " elle-même. L'Homme nouveau est de retour; seulement, c'est maintenant un fonctionnaire du FMI 29 .

L'Empire apparaît donc comme un empire totalitaire et fermé. Rien ne peut lui échapper. Il déclare qu'il n'y a pas d'alternative, et il possède

27 Hugo Assmann cite, en ce sens, Roberto CAMPOS : « En toute rigueur, personne ne peut opter directement pour les pauvres. L'option qu'il faut faire passe par l'investissement qui crée des emplois pour les pauvres (Hugo ASSMANN, Economía y religión, San José, DEI, 1994, p. 101.)

28 Voir, par exemple : Peter G. MOLL, « Liberating Liberation Theology : Towards Independence from Dependency Theory ", Joutnal of Theology for Southern Africa, Mars 1992; Roger HAIGH, S.J., An Alternative Vision : an Interpretation of Liberation Theology. New York, Paulist Press, 1985; Amy L. Sherman, Preferential Option. A Christian and Neoliberal Strategy for Latin America's Poor. Grand Rapids (MI), Eerdmans, 1992. Sur certe sacralisation du marché dans le contexte de la modernité, voir Julio de SANTA ANA, « Teologia o modernidade ", dans Antonio Silva (dir.), América Latina: 500 anos de evangelização : reflexões teológico-pastorais. São Paulo, Paulinas, 1990. 
le pouvoir de réprimer avec tant de dureté n'importe quelle tentative de recherche de changement qu'en effet, il semble mieux valoir de ne pas s'y essayer. Quand le châtiment est plus grand que ce qu'on pourrait obtenir en cherchant des alternatives, il est préférable de ne pas les chercher. Dans une telle situation, le pouvoir dicte ce que dira la réalité. Entre le pouvoir et la réalité s'établit un circuit à l'intérieur duquel la réalité confirme tautologiquement les thèses du pouvoir ${ }^{30}$.

Cette condition de l'Empire totalitaire et fermé, c'est ce que plusieurs théologiens de la libération perçoivent maintenant, dans la tradition judéo-chrétienne, comme la situation apocalyptique. Dans une telle situation, il n'y a pas d'issue en vue et il ne peut y avoir de projets concrets de changement. On recommence à lire l'Apocalypse de saint Jean et en discuter. En ce sens traditionnel, l'Apocalypse est une révélation. L'Apocalypse révèle que, face à l'Empire totalitaire et fermé, il existe une alternative, même si on ne sait pas laquelle. Le pouvoir totalitaire de l'Empire révèle

30 Hannah Arendt décrit ce circuit d'une manière magistrale : * L'affirmation voulant que le métro de Moscou soit le seul existant dans le monde est un mensonge tant et aussi longtemps que les bolcheviques ne prennent pas le pouvoir pour détruire tous les autres métros. En d'autres mots, la méthode de prédiction infaillible, plus que n'importe quelle autre méthode de propagande totalitaire, révèle indirectement son objectif ulrime de conquête mondiale étant donné que c'est seulement dans un monde complètement sous son contrôle que le dominateur totalitariste pourrait possiblement transformer en réalité tous ses mensonges et arriver à réaliser toutes ses prophéties * (Hannah ARENDT, Los origines del totalitarismo. Madrid, Taurus, 1974, p. 435). "Alors toute discussion au sujet du bien fondé ou de la fausseté de la prédiction d'un dictateur totalitaire devient aussi chimérique que de discuter avec un assassin professionnel pour savoir si sa future victime est morte ou vivante, érant donné qu'en tuant la personne en question, l'assassin peut fournir immédiatement la preuve de la véracité de sa déclaration" (Ibid.). Dans une entrevue, Camdessus décrit ce mécanisme à partir du FMI : (Question) : «Quel sera le coût social des mesures prises pour remettre en ordre les finances publiques? " (Réponse) : "Posons plutôt la véritable question : quel serait le prix à payer pour le peuple du Costa Rica s'il n'acceptait pas d'ajuster ses structures? Le prix pourrait être l'interruption du financement intérieur, la réduction des investissements, la suspension d'un accord de renégociation de la detre, l'interruption des importations. Le coût serait la récession... Notre position n'est pas exactement de recommander ou d'imposer des mesures. Notre position en est une de dialogue... Mais le fait que les objectifs n'ayant pas été atteints, nous devions suspendre les versements, ne constituerait pas une punition, mais une réalité à laquelle le pays doit faire face en adoptant ses policiques. Nous recommencerions alors aussitôt les versements ("Entrevista a Michel Camdessus, director-gerente del FMI », La Nación, San José, 5 mars 1990). 
sa faiblesse sans que, néanmoins, sa chute soir vue comme le résultat d'une action intentionnelle. Le nom de cet empire est Babylone.

Une telle lecture de l'Apocalypse conduit à l'analyse des textes apocalyptiques connus, mais aussi à l'analyse de leur contexte économique, social et politique. On redécouvre le fait que ces textes sont apparus dans des situations historiques semblables à la nôtre. Le croyant y fait face à un empire ne lui laissant aucune issue; il persiste cependant à dire qu'il doit y avoir une quelconque porte de sortie.

"Apocalypse " n'a pas le sens, ici, d'une catastrophe. Le titre du film "Apocalypse now " n'indique d'aucune façon le sens dont il s'agit dans le cas présent. En tant que révélation, l'Apocalypse rappelle que le monstre est un géant aux pieds d'argile dont la chute laissera l'avenir ouvert à la réalisation d'alternatives ${ }^{31}$. Cette lecture de l'Apocalypse, telle qu'elle apparaît maintenant dans la théologie de la libération, n'est pas non plus directement comparable à celle que fait le fondamentalisme chrétien américain. Dans cette dernière, l'Apocalypse est une fois de plus la catastrophe qui se produit par la volonté de Dieu, au sens d'une loi inexorable de l'histoire. Le monde est condamné à périr et, son salut, c'est un dieujuge qui le réalisera quand l'histoire elle-même sera consommée. Bien sûr, cette loi de l'histoire est celle de la catastrophe de l'histoire humaine. Il s'agit d'un fondamentalisme qui a la prétention d'écrire aujourd'hui l'histoire de demain. Les Présidents Reagan et Bush s'inscrivirent publiquement dans cette métaphysique de l'histoire ${ }^{32}$. De même, la récupération

31 Le texte qui décrit peut-être le mieux la situation apocalyptique est celui du prophète Daniel qui a vécu au II ${ }^{e}$ siècle avant Jésus-Christ : «Tu as eu, ô roi, une vision. Voici : une statue, une grande statue, extrêmement brillante, se dressair devant toi, terrible à voir. Cette statue, sa tête était d'or fin, sa poitrine et ses bras étaient d'argent, son ventre et ses cuisses de bronze, ses jambes de fer, ses pieds partie fer et partie terre cuire. Tu regardais: soudain une pierre se détacha, sans que main l'eût touchée, et vint frapper la statue, ses pieds de fer et de terre cuite, et les brisa. Alors se brisèrent, tout à la fois, fer et terre cuite, bronze, argent et or... Er la pierre qui avait frappé la statue devint une grande montagne qui remplit toute la terre " ( $\left.D_{n} 2,31-35\right)$

32 Le livre de Pentecost, déjà cité plus haut, est bien intitulé Evento del porvenir. De la même façon que l'Académie des sciences de Moscou connaissait les lois inexorables de l'avenir, ces fondamentalistes les connaissent également. Mais, alors que l'Académie des sciences croyait tout de même en un avenir meilleur, le fondamentalisme actuel croir que la perte de l'humanité est déjà décidée à l'avance. Dès le premier jour de la Guerre du Golfe, le Président Bush est apparu à la télévision avec un des prédicareurs fondamentalistes américains, Billy Graham, pour implorer avec lui la bénédiction de Dieu sur cette guerre. 
que Camdessus essaie de faire de la théologie de la libération est parfaitement compatible avec une telle vision du monde.

La lecture actuelle de l'Apocalypse que font quelques théologiens de la libération, ne correspond à aucune de ces interprétations. L'Empire totalitaire et fermé d'aujourd'hui comme l'étaient les empires romain, grec ou babylonien, c'est une véritable Babylone, et donc un géant aux pieds d'argile. Il s'écroule et quelle que soit la raison de sa chute, celle-ci ne peut résulter d'un acte humain volontariste. Il est trop fort pour cela. Il tombe à cause d'effets non intentionnels, résultat de sa propre toute-puissance. Mais il n'y a pas de loi métaphysique de l'histoire qui le fasse tomber. Il tombe parce que ... « soudain une pierre s'est détachée, sans que main l'eût touchée, vint frapper la statue, ses pieds de fer et d'argile et les brisa 33 ".

Bien sûr, la situation apocalyptique ne provoque pas uniquement le genre littéraire que nous appelons apocalyptique. Elle donne aussi lieu à un autre genre qui lui est jumeau : la littérature sapientielle, qui a toujours été l'autre face de la littérature apocalyptique. Un des grands témoignages de cette littérature, repris de nos jours par la théologie de la libération, est le livre de la Bible qu'on appelle l'Ecclésiaste et qui a été écrit au troisième siècle avant Jésus-Christ. Il exprime un sens plutôt tragique de la vie face à un empire inexpugnable et dévastateur. Une lamentation sur la perte du sens de la vie y prévaut, combinée à l'écho lointain de l'espérance détruite. Il s'agit d'une perception de la vie qui a beaucoup en commun avec certains courants de la postmodernité actuelle. Comme certains théologiens reviennent à la lecture de l'Apocalypse, on voit aussi apparaître cette problématique, aujourd'hui, dans la théologie de la libération 34 .

33 Voir Pablo Richard, « El pueblo de Dios contra el imperio. Daniel 7 en su contexto literario e histórico », RIBLA 7 (1990); Apocalipsis. Reconstrucción de la esperanza. San José, DEI, 1994; Carlos MESTERS, El Apocalipsis : la esperanza de un pueblo que lucha. Santiago de Chile, Rehue, 1986; Ricardo FOULKES, El Apocalipsis de San Juan. Una lectura desde América Latina. Buenos Aires, 1989; Christopher RowlaND, Radical Christianity : A Reading of Recovery. New York, Orbis Books, 1988; Juan SNOEK, Rommie N AUTA, Daniel y el Apocalipsis. San José, DEI, 1993.

34 Voir Elsa TAMEZ, « La razon utopica de Qohélet », Pasos 52 (mars-avril 1994), pp. 9-23. 


\section{Le défi pour la théologie de la libération. L'irrationalité du « rationalisé »}

Comme nous l'avons vu de près avec la théologie du FMI, on est passé de la négation de la théologie de la libération à la construction d'une antithéologie de la libération. Cette anti-théologie apparait clairement comme une inversion de la théologie de la libération.

De nouveau ressort le fait que ce n'est pas au niveau d'une discussion strictement théologique que peuvent se distinguer ces deux théologies opposées. Sur ce plan, la théologie de la libération ne se distingue pas clairement de l'anti-théologie promue actuellement par le FMI. On débouche, en apparence, sur une situation où le conflit cesse d'être théologique. Le conflit paraît porter sur l'application d'une théologie que partageraient les deux parties. La théologie de l'Empire - la théologie du FMI est la théologie de l'Empire - s'est appropriée les éléments-clés de la théologie de la libération: l'option préférentielle pour les pauvres et l'espérance du Royaume de Dieu incarnée dans l'orthopraxie. C'est, du moins, ce qui paraît se produire.

Le problème que nous avions abordé au début de cet article revient maintenant à un autre niveau. Nous y avons analysé le fait que, durant les années 60 et 70 , la théologie de la libération avait surgi de telle manière que le conflit avec la théologie institutionnalisée n'apparaissait pas d'abord comme un conflit religieux, étant donné qu'aucun dogme religieux n'était remis en question. Le conflit apparaissait comme un différent sur les implications concrètes d'une foi commune. L'option préférentielle pour les pauvres et l'incarnation du Royaume de Dieu dans le monde économico-politique représentaient des instruments de cette mise en pratique de la foi. En tant que théologie prenant en compte la concrétude, la théologie de la libération prêtait à être interprétée de façon conflictuelle.

Actuellement, au contraire, c'est la théologie de l'Empire elle-même qui assume ces positions. Celles-ci ne servent pas, bien sûr, à donner un caractère concret à la foi. La théologie de l'Empire se dit d'accord avec l'option préférentielle pour les pauvres et avec l'incarnation économicosociale du Royaume de Dieu. Et elle se présente même comme l'unique voie réaliste à la hauteur de ces exigences. La théologie de l'Empire fausse, bien entendu, les principes de base de l'option pour les pauvres de la théologie de la libération. Du point de vue de cette demière, cette option est la conséquence d'une reconnaissance mutuelle entre les sujets humains. Le pauvre est le signe d'une perte de cette reconnaissance, indication que toute relation sociale humaine se trouve dénaturée. La théologie de l'Empire, au contraire, ne peut envisager le pauvre autrement que 
comme un objet pour les autres, c'est-à-dire pour ceux qui ne sont pas pauvres.

L'option pour les pauvres ne peut plus alors s'identifier avec aucune concrétisation de la théologie de la libération. La question qui se pose n'est plus, maintenant, que celle du réalisme de sa concrétisation. Aucune foi préconçue ne peut apporter de réponse. On peut décider de la vérité d'une position sans recourir aux sciences empiriques, en particulier aux sciences économiques. Ce sont elles qui décident. Elles se transforment alors en porteuses du critère de vérité au sujet des théologies. En effet, avec l'économie politique néo-libérale en main, l'option pour les pauvres se transforme en option pour le FMI. Du point de vue d'une économie politique critique, au contraire, elle se transforme en l'exigence d'une société alternative dans laquelle tous auraient leur place. La théologie en tant que telle ne peut décider. Ce sont les résultats de la science qui décident à propos du contenu concret de la théologie.

Voilà pourquoi les tentatives de récupération de la théologie de la libération obligent celle-ci à développer de nouvelles problématiques. Pour pouvoir continuer à soutenir l'option pour les pauvres en des termes qui respectent le pauvre comme sujet - ce qui est particulier à la théologie de la libération - cette option doit être liée d'une façon beaucoup plus déterminée à la reconnaissance mutuelle entre les sujets en tant qu'êtres corporels et soumis à des besoins.

Cela conduit à la nécessité de développer la théologie de la libération dans deux directions en particulier. La première touche à la critique de l'économie politique néo-libérale et de son utopisation de la loi du marché. La deuxième concerne la tradition chrétienne d'une théologie critique de la loi. Ces deux pistes constituent ensemble l'espace d'un débat qui se pose souvent, aujourd'hui, en termes de relation entre l'économie et la théologie. Ce débat constate la pertinence de l'analyse économique pour le discernement du contenu de la foi et dépasse le point de vue qui ne considérait l'économie que comme un espace d'application de la foi.

Dans le premier volet, celui de la critique de l'économie politique néo-libérale, l'argument pourrait se résumer par la phrase suivante: la rationalisation par la compétitivité et la productivité (rentabilité) révèle la profonde irrationalité du rationalisé. L'efficacité n'est pas efficace. En réduisant la rationalisation à la rentabilité, le système économique actuel tombe dans l'irrationalité. Il déchaîne des processus destructeurs qu'il ne peut contrôler à partir des paramètres de rationalité qu'il avait choisis. L'exclusion d'un nombre croissant de personnes du système économique, la destruction des bases naturelles de la vie, la distorsion de toutes les rela- 
tions sociales et, par conséquent, des relations marchandes elles-mêmes, sont le résultat non-intentionnel de cette réduction de la rationalité à la rentabilité. Les lois du marché du capitalisme total détruisent la société elle-même et son environnement naturel. En absolutisant ces lois par la médiation du mythe de l'automatisme du marché, ces tendances destructrices sont devenues incontrôlables et se sont transformées en menace pour la survie même de l'humanité.

Cette critique débouche sur une analyse de la rationalité qui inclut précisément l'irrationalité du rationalisé. L'enjeu est de développer le concept d'un circuit naturel et social de la vie humaine qui englobe et se soumette la rationalité instrumentale (moyen-fin) qui est sous-jacente au calcul de la rentabilité. Cela exclut la totalisation néo-libérale de la loi du marché, pour intégrer les relations du marché dans la vie sociale. La politique néo-libérale, au contraire, traite le marché comme l'élément constituant de toutes les relations sociales, débouchant ainsi sur sa politique du capitalisme total ${ }^{35}$.

Avec une analyse de ce type, la théologie de la libération relève le défi de se confronter avec la pensée de Marx ${ }^{36}$. C'est ce qui arrive même si elle ne le fait pas délibérément. La pensée de Marx est le grand corps théorique existant qui a précisément surgi pour faire la critique de l'irrationalité du rationalisé. En affrontant, aujourd'hui, ce problème sur le plan théorique, n'importe quel effort de conceptualisation élabore des réflexions proches de celles que Marx, le premier, a développées. Voilà pourquoi, même si quelqu'un ne part pas de Marx, il arrive, sans le chercher, à ses concepts ou s'en approche. Et dans ce dialogue avec la pensée de Marx, se développe une critique profonde de celle-ci, qui est fondamentale pour les réflexions de la théologie de la libération. Cette critique concerne l'espérance marxiste : pouvoir solutionner les problèmes du capitalisme total par un dépassement total du capitalisme. Le marxisme débouche sur une totalisation analogue à celle que nous vivons actuellement avec la totalisation néo-libérale du capitalisme. La théologie de la libération doit dépasser les totalitarismes si elle veut contribuer

Helio Gallardo, « Radicalidad de la teoría y sujeto popular en América Latina », Pasos Especial 3 (1992); Franz J. HINKELAMMERT, "La lógica de la expulsión del mercado capitalista mundial y el proyecto de liberación », Pasos Especial 3 (1992), pp. 3-21.

36 Voir Enrique DUSSEL, La producción teórica de Matx. Un comentatio a los *Grundrisse*. México, Siglo XXI, 1985; Hacia un Marx desconocido. Un comentario de los Manuscritos del 61-63. México, Siglo XXI, 1988. 
réellement à la constitution d'une foi nouvelle. Cependant, une fois qu'on a critiqué ces totalitarismes, les conceptualisations de la critique de l'irrationalité du rationalisé restent indispensables pour pouvoir constituer un concept adéquat de la rationalité de l'action humaine. La théorie de l'action rationnelle, comme l'a développée Max Weber, ne va pas plus loin que cette réduction de l'action rationnelle à ses expressions en termes de rationalité instrumentale, c'est-à-dire à ce qui est susceptible d'être mesuré en termes de rentabilité.

Cela nous amène au deuxième volet du développement nécessaire de la théologie de la libération dans le monde d'aujourd'hui. La critique de l'irrationalité du rationalisé doit être exprimée en des termes proprement théologiques. Cela se produit justement par la récupération d'une grande tradition théologique de critique de la loi, qui commence dans les Écritures et qui trouve sa première élaboration théologique chez Paul de Tarse, surtout dans l'Épître aux Romains. De fait, c'est la première élaboration de la critique de l'irrationalité du rationalisé qu'on trouve dans la pensée chrétienne ${ }^{3}$.

Les théologiens de la libération qui reprennent cette théologie de Paul font ressortir deux éléments-clés de sa critique de la loi. D'une part, Paul montre que la loi, en tant que loi d'observance, conduit à la mort ceux qui s'y soumettent ou qui sont obligés de l'observer au-delà de toute autre considération. La loi, qui devrait servir à la vie, mène dans ce cas à la mort. Paul parle de la "loi d'observance" (ley de cumplimiento) qui mène à la mort. La loi, ici, peut être n'importe quelle loi, autant la loi juive du type de celle que Paul connait par la tradition pharisaïque, que la loi romaine. Par conséquent, l'Étar de droir qui apparaît avec l'empire romain pour la première fois dans l'histoire, ne représente pas la plus haute expression de l'humanité, mais bien plutôt une menace. La loi ne sauve pas par l'observance de celle-ci. D'autre part, Paul ne conçoit pas le péché comme une infraction à la loi. Le péché dont il s'agit dans la vision de Paul est celui qui est commis dans l'observance la loi et pour qu'elle soir observée. Les infractions à la loi sont secondaires. Par conséquent, le péché c'est d'aboutir à la mort en observant les prescriptions de la loi. Donc, le péché est commis même avec la bonne conscience d'être en train

37 Le livre le plus remarquable sur ce sujet est celui d'Elsa TAMEZ, Contra toda condena. La justificación por la fe desde los excluidos. San José, DEI, 1991. Voir Richard SHAULL, La Reforma y la teologia de la liberación, San José, DEI, 1993 et aussi Franz J. HINKELAMMERT, Las armas ideológicas de la muerte. San José, DEI, 1981 (2 édition révisée et augmentée, avec une introduction de Pablo Richard et Raúl Vidales). 
d'observer la loi. On trouve encore des traces de cette réflexion dans le dicton du Moyen Âge européen: Summa lex, maxima iniustitia. Ou dans cet autre qui lui est contraire, mais qui a un sens ironique : Fiat iustitia, pereat mondus (Que la loi soit observée, le monde dût-t-il en périr).

Cela mène à l'analyse de la sacrificialité comme résultat de la loi. La loi, quand elle est conçue de manière totalisante, exige des sacrifices humains. On avait une vive conscience de cela au tout début du christianisme. Tous les évangiles, par exemple, insistent sur le fait que c'est en observant la loi qu'on a tué Jésus, en accomplissant la loi. Dans cette mesure même, il n'y a pas de coupables personnels de cette mort. C'est sa relation avec la loi qui est à son origine. On comprend ainsi mieux que la théologie conservatrice ait, plus tard, préféré culpabiliser les Juifs. Elle se devait de le faire pour échapper aux conséquences de la théologie de la critique de la loi qui était complètement incompatible avec les aspirations du christianisme au pouvoir impérial ${ }^{38}$.

Ce fair explique pourquoi, précisément, la théologie de la critique de la loi de Paul, son affirmation, sa négation, son inversion et sa falsification, ont été le fil conducteur de l'histoire du christianisme, et par conséquent de l'Occident. Son écrit majeur est l'Épître aux Romains. Ce n'est pas du tout un livre "théologique ", au sens que l'on donne à cette division de l'université moderne qu'on appelle faculté de "théologie ». Cet écrit analyse quelle est la clé de l'empire romain, au temps de Paul, et il le fait d'un point de vue théologique. L'Épître est aussi décisive pour la philosophie et la pensée politique qu'elle peut l'être pour la pensée théologique. Pour les philosophes, cependant, elle est taboue. On n'en fait pas mention dans les histoires de la philosophie, même si plus de mille cinq cents ans de la pensée occidentale, y compris la pensée philosophique, ont tourné autour de cette pensée. L'Épître aux Romains fut décisive au temps de la Réforme, surtout pour Martin Luther. Voilà pourquoi on la retrouve à la base de l'éthique protestante et de la transformation de celle-ci dans l'esprit du capitalisme. Elle a aussi été très importante dans l'émergence de la théologie moderne avec le livre de Karl Barth sur l'Épître aux Romains. Aujourd'hui, elle redevient décisive pour la théologie de la libération. C'est l'un des livres les plus subversifs de l'histoire. L'une des

Voir Franz J. HinkelammeRT, La fe de Abraham y el Edipo occidental. San José, DEI, 1991 ( $2^{\mathrm{e}}$ éd. augmentée); Sacrificios humanos y sociedad occidental : Lucifer y la Bestia. San José, DEI, 1991; Jorge PIXLEY, "La violencia legal, violencia institucionalizada, la que se comete creyendo servir a Dios », RIBLA 18 (1994), pp. 7 17. 
rares personnes qui s'en soit rendue compte est Nietzsche. Mais Nietzsche l'a fait seulement parce qu'il avait choisi Paul comme son ennemi de prédilection.

La critique paulinienne de la loi est une critique des lois «justes» que Paul découvre surtout dans les lois de Dieu données sur le Sinaï. Toutefois, par rapport à sa critique de la loi, ces commandements ne sont pas non plus, en eux-mêmes, des lois justes. Toute loi, selon Paul, tue si elle est traitée comme une loi d'observance. De son point de vue, cela vaut aussi pour la "loi de Dieu". L'injustice réside dans la forme générale de la loi. Par conséquent, la justice de Paul ne réside pas dans la loi, mais plutôt dans la relation à celle-ci. Le sujet est souverain face à la loi, afin de pouvoir la relativiser dans tous les cas où son observance tuerait.

Il y a une différence catégorique entre cette critique de la loi de Paul et la tradition libérale. La tradition libérale cherche des lois justes. Elle croit les avoir trouvées en soutenant que la loi est juste quand ceux qui ont le devoir de lui obéir incluent aussi ceux qui, comme citoyens, sont également les législateurs. Pour cette tradition, cette loi est " loi de Dieu ": vox populi, vox dei. Par conséquent, le résultat libéral c'est que la loi démocratique est une loi juste. Alors, un Érat de droit basé sur certe loi est un État juste.

Pour Paul, il n'y a pas d'État de droit au sens que l'entend l'idéologie dont nous parlons ici, et selon laquelle l'État de droit aurait des lois justes, avec certe conséquence que le citoyen a le devoir de leur obéir sans possibilité d'effectuer un discernement ou de leur résister. Max Weber définit cette démarche comme "la légitimité par la légalité ». Elle est incompatible avec la position de Paul, qui en est une de discernement. D'un point de vue paulinien, l'État de droit libéral est un État injuste, parce que c'est un État totalitaire. La position de Paul implique le droit à la résistance, la position de Weber dénie ce droit.

Actuellement, on perçoit clairement que se prépare un holocauste du Tiers Monde. S'il se produit, il aura été perpétré par des États de droit et à l'intérieur des strictes limites des États de droit ${ }^{39}$. C'est précisément ce que révèle le fait que l'État de droit ne garantisse d'aucune manière le respect de la justice. La résistance humanise l'État de droit, et là où la

39 Voir Jean-Christophe RUFIN, L'empire et les nouveaux barbares. Rupture Nord-Sud. Paris, Jean-Claude Latrès, 1991, 255 p. Aussi, Hans Magnus Enzensberger, Aussichten auf den Bürgerkrieg. (Perspectives de la guerre civile), Frankfurt Main, Suhrkamp, 1993. 
résistance est réprimée avec succès ou est empêchée, l'État de droit se transforme en un Moloch. C'est pour cela que l'État de droit, comme démocratie, et le totalitarisme sont compatibles 40 . On peut penser aussi au recouvrement de la dette extérieure du Tiers Monde. N'est-ce pas là un crime qui est commis "en observant la loi "? C'est l'État de droit luimême qui le commet. Quand Paul parle du péché, il se réfère à ces crimes qui se commettent "en obéissant à la loi ». Les transgressions de la loi l'intéressent à peine ${ }^{41}$. Il s'intéresse surtout aux crimes qui se commettent avec bonne conscience et en croyant ainsi servir Dieu, l'humanité et les pauvres.

C'est dans ce sens que la théologie de la critique de la loi développe le problème de l'irrationalité du rationalisé. À cause de cela, les théologiens de la libération peuvent reprendre cette théologie en la mettant en relation avec la loi du marché. D'un côté, en étant considérée comme une loi qui sauve lorsqu'on lui obéit, la loi du marché conduit à la mort, y compris celle de l'humanité. D'autre part, il y a un péché qui est commis quand on obéit à la loi du marché avec la bonne conscience d'être en train d'obéir à loi suprême de l'humanité. Survient alors la liberté chrétienne dans le sens où Paul l'a définie, une liberté qui est souveraine face à la loi. Les sujets libres sont libres dans la mesure où ils sont capables de relativiser la loi en fonction des besoins de leur propre vie. Le liberté n'est pas dans la loi mais bien dans la relation des sujets avec la loi. Face à la loi du marché, la liberté consiste à pouvoir soumettre celle-ci, quitte à commettre des infractions contre elle si les besoins des sujets le commandent. La reconnaissance mutuelle entre les sujets corporels et soumis à des besoins implique, de façon non négociable et sans substitution possible, qu'on reconnaisse la relativité de toute loi en regard de cette reconnaissance. En nous reconnaissant mutuellement comme sujets, nous nous reconnaissons comme souverains face à la loi. La loi ne vaut que dans la mesure où elle ne nous interdit pas cette reconnaissance mutuelle.

On peut maintenant reprendre l'option pour les pauvres selon une perspective et un sens que la théologie de l'Empire n'acceptera jamais. La reconnaissance mutuelle entre des sujets corporels et soumis à des besoins de base implique l'option pour les pauvres et, par le fait même, la souve-

Dans la littérature sur le totalitarisme, il n'y a que Hannah Arendt qui rende compte de ce fait. Voir Hannah ARENDT, op. cit.

41 Voir Franz J. HINKelammert, La deuda externa de América Latina: el automatismo de la deuda. San José, DEI, 1988; Democracia y totalitarismo. San José, DEI, 1987. 
raineté du sujet humain face à la loi. Sans cette souveraineté, il ne peut y avoir ni reconnaissance mutuelle entre les sujets ni option pour les pauvres. À partir d'une reconsidération de ce concept, apparaît du même coup une reconsidération du concept de Royaume de Dieu ${ }^{42}$.

Voilà pourquoi la théologie de la libération ne nie pas seulement l'absolutisation de la loi du marché dans le "capitalisme total ", mais également n'importe quelle loi métaphysique de l'histoire. L'absolutisation de la loi - c'est-à-dire sa transformation en loi métaphysique de l'histoire - est une totalisation qui, à la fin, débouche sur le totalitarisme. Sa devise est toujours celle de la "fin de l'histoire " et de la négation de toutes les alternatives 43 .

Avec ce résultat, la théologie de la libération en arrive à une critique de la modernité et non uniquement du capitalisme. Elle en arrive à la constatation d'une crise de la société occidentale elle-même. Elle n'en est pas pour autant postmoderne. Les postmodernes se gardent bien de faire l'analyse de la loi du marché en tant que loi métaphysique de l'histoire. Ils attaquent de toutes parts les lois métaphysiques de l'histoire, en particulier celles qui ont été véhiculées dans le socialisme historique. Mais la loi du marché, comme unique cas actuel d'une imposition d'une loi métaphysique de l'histoire, ils ne la mentionnent même pas. Ils dissimulent la loi métaphysique de l'histoire en vigueur aujourd'hui au nom de la critique d'autres lois de l'histoire qui ont eu cours dans le passé.

42 Voir Jung Mo SUNG, Economía. Tema ausente en la teologia de la liberación. San José, DEI, 1994.

43 Hugo ASSMANN, «Teología de la liberación : mirando hacia el frente ", Pasos 55 (septembre-octobre 1994), pp. 1-9; Franz J. HINKELAMMERT, « ¿Capitalismo sin alternativas? Sobre la sociedad que sostiene que no hay alternativa para ella », Pasos 37 (septembre-octobre 1991), pp. 11-24. 